\title{
VARIA
}

\section{FIGURES DE LA DOMINATION : LES IMPLICATIONS ÉPISTÉMIQUES ET POLITIQUES DE L'APPROCHE SOPHISTIQUE}

\begin{abstract}
Marie-Joseph BERTINI ${ }^{1}$
Il fut et demeure le premier des medias, ce langage que Mc Luhan décrivait comme une «technologie du prolongement humain» (1968). Figure essentielle de la domination, le langage, et singulièrement la parole, véhiculent et façonnent les valeurs et les lois qui ordonnent sociétés et cultures. A cette dimension politique de la parole organisée en discours, s'articule une dimension polémologique moins explorée, dont l'analyse permet de montrer combien la rhétorique, entendue comme l'art du discours, et l'art de la guerre - celle subtile et rusée de l'argumentation - sont intimement liés. Si l'on connaît mieux aujourd'hui les formes et les vertus de l'argumentation $n o b l e^{2}$, on ignore encore trop comment l'élaboration des modalités de l'argumentation spécieuse autorisa le développement d'une véritable pensée communicationnelle, redéfinissant d'une manière particulièrement originale la dynamique du savoir et des relations sociales.
\end{abstract}

1 Maître de Conférences en Sciences de l'Information et de la Communication, Chercheur au Laboratoire I3M (Information, Milieux, Médiations, Médias), Faculté des Lettres, Arts et Sciences Humaines, Université de Nice-Sophia Antipolis.

2 Voir à cet effet les numéros 15 et 16 de la revue Hermès, Argumentation et rhétorique, I et II, 1995.

Recherches en communication, $\mathrm{n}^{\circ} 24$ (2005). 


\section{La sophistique, première théorie de la communication}

Contrairement à ce que l'on pourrait être tenté de croire, l'idée selon laquelle la maîtrise des codes du langage pouvait procurer un pouvoir réel est très ancienne. Elle trouve l'une de ses premières et mémorables incarnations dans la démarche de quelques esprits originaux qui, dès le $\mathrm{V}^{\circ}$ siècle avant Jésus-Christ, s'illustrèrent sous l'épithète de Sophistes.

L'ampleur d'une telle démarche et les implications qui en résultent font des Sophistes les premiers théoriciens de la communication. Véritable mouvement de contestation linguistique, rhétorique et philosophique, la sophistique répandit dans le monde grec, par ailleurs si épris de mesure et de vérité (alétheia), un peu de ce cynisme iconoclaste et corrosif dont notre modernité sera si fort empreinte. Ils n'hésitèrent pas à troquer le réel contre l'apparent, le vrai contre le vraisemblable, l'unique contre le multiple, l'univoque contre l'équivoque, la tentation pérenne de l'absolu contre les vertiges du relatif.

Les règles de la communication, et le travail sur le langage en particulier, leur fournirent un champ d'expériences privilégiées. Affranchissant ce dernier de la domination polarisante du vrai, ils épanouirent ses diverses modalités en faisant appel à de nouveaux registres, jugés alors peu orthodoxes. A la fonction cohésive du langage, ils substituèrent une fonction subvertive, renversant les valeurs établies et les idées reçues de leur temps, engageant les mots non plus au service du vrai idéal, mais des passions et des intérêts humains.

De fait, la sophistique élabore les prémisses d'une véritable pragmatique de la communication. Pour les Sophistes, la langue constitue le produit d'une pure convention. Ni les termes usités, ni les relations qu'ils entretiennent dans le discours ne revêtent un caractère hiératique et immuable. Le scepticisme sophistique imprègne toutes les catégories de la connaissance. A la fois gnoséologique et pratique, il est porteur d'une nouvelle définition de la rationalité, très proche par certains aspects de l'Epistémé contemporaine.

A partir des Sophistes, quelque chose d'exceptionnel est à l'œuvre au niveau du langage. Celui-ci n'est plus cet organon qui sert à dire l'Etre, il ne répond plus à une exigence ontologique, mais à une nécessité intersubjective et polémique, en devenant l'instrument privilégié de la rencontre frontale avec l'autre. La rhétorique regagne ici le terrain abandonné par l'ontologie. Un tel processus alimente un foyer théo- 
rique à partir duquel l'ensemble des pratiques communicationnelles vont rayonner. Il constituera le creuset durable d'un relativisme à la fois pratique et connaissant.

\section{Le relativisme est un humanisme}

Contrairement aux thèses de l'ontologie platonicienne, la réalité ultime, c'est-à-dire l'Etre, n'est pas conçue par les Sophistes comme une immuable unité. Elle ne saurait être appréhendée d'aucune manière en raison de son caractère de profonde instabilité et de sa multiplicité. «Touchant les dieux, je ne suis pas en mesure de savoir s'ils existent ou s'ils n'existent pas», écrit Protagoras d'Abdère', l'un des maîtres de cette révolution copernicienne. L'impossibilité de dépasser l'horizon des phénomènes, c'est-à-dire des apparences, induit un relativisme à la fois spéculatif et opératoire. C'est la raison pour laquelle l'ambition sophistique ne consiste pas à mettre en place une pratique communicationnelle qui ferait fond sur la négation du savoir et de la morale, mais à démontrer que savoir et morale ne corrèlent aucune donnée immuable de la nature, puisqu'aussi bien, ils sont le résidu d'une convention humaine. Tel est le sens de la formule fameuse de Protagoras: «L'homme est la mesure de toutes choses» ${ }^{2}$.

Nous déchiffrons ainsi le monde à travers une double grille perceptive et réflexive qui trouve sa légitimité dans la dialectique des intersubjectivités.

D'où la radicalité de cette analyse qui va à l'encontre des certitudes post-modernes (Baudrillard) : l'apparence n'est pas ici le simulacre des choses, mais leur être véritable, réfléchi dans les activités humaines disparates.

Phénoménologues avant la lettre, les Sophistes postulaient qu'il n'y a pas de réalité secrète, dissimulée, des choses, pas d'arrière-monde tapi dans l'ombre des phénomènes. Ils suppriment, ce faisant, la dichotomie opposant l'être et le paraître, plate-forme de l'appréhension occidentale du monde. L'apparence n'est plus ici une manifestation inconsistante de l'être, voire son négatif. Elle est l'être lui-même qui n'est rien d'autre que ce qu'il paraît être à la seule instance capable de le penser et de l'ordonner en éléments hiérarchisés, à savoir la conscience

1 «Sur les dieux», in Eusèbe de Césarée, Préparation évangélique, XIV, III, 7, Cerf, 1974.

2 Platon, Thééthète ou De la science, Les Belles Lettres, coll. Guillaume Budé, 1969. 
humaine. Celle-ci procède par arte fact, c'est-à-dire par art ou artifice, ce qui revient à peu près au même dans cette perspective.

On le voit, ce relativisme est un humanisme. On saisit, dès lors, les raisons qui amenèrent Platon à ruiner les thèses sophistiques qui contrevenaient par trop à un idéal apollinien d'ordre et de mesure fondé sur la permanence et l'objectivité dont le philosophe se fit le héraut. Le Sophiste demeura à jamais pour lui, cet ennemi héréditaire qui porte au sein de la communauté intersubjective le coup fatal de la contradiction, du cynisme, de l'opinion, de l'imitation et du simulacre réunis ${ }^{1}$. Ainsi la sophistique déploie-t-elle les artifices communicationnels à l'intérieur de l'hypothèse regulatrice d'une humanité devenue le seul champ référentiel possible.

En ce sens, ses implications, tant théoriques que pratiques, sont fondamentales.

Les Sophistes ne sont-ils pas les premiers à démontrer ainsi que les valeurs et les principes reconnus autour desquelles s'articulent les sociétés, sont susceptibles de varier en fonction des individus et des sociétés qui les déterminent ? Plus encore, ils mettent en évidence la nature de ce qui fonctionne comme valeur, sur le plan éthique aussi bien qu'épistémologique, puisque selon eux, ce qui fonde une valeur ce n'est pas sa véracité, mais le fait qu'elle soit acceptée comme telle, qu'elle s'enracine dans un consensus.

\section{La construction politique et sociale du savoir}

La redécouverte de cette dimension éminemment sociale du savoir, en particulier scientifique, sera l'une des conséquences majeure de la réflexion engagée par les nouveaux anthropologues des sciences ${ }^{2}$. Cette vision pragmatique de la rationalité ruine l'idée d'un critérium de rationalité a priori, et constitue la science en une démarche picaresque, entreprise aventurière et audacieuse travaillant avec des contraintes stratégiques et tactiques mettant en lumière les nécessaires contextualisations des pratiques qui se réclament d'elle.

Ainsi, précédant le quasi immobilisme ontologique des théories platoniciennes, la sophistique met-elle en scène les concepts centraux d'intérêt et d'efficacité. A la définition apollinienne de la communica-

1 Cf. Le Sophiste, Les Belles Lettres, coll. Guillaume Budé, 1969.

2 Cf. Les travaux de Bruno Latour sur l'anthropologie des sciences. 
tion, lisse, ancrée dans l'idée de permanence, s'oppose donc une pragmatique pré-moderne empreinte d'un relativisme foncier qui témoigne d'une certitude subjective, et non d'un pessimisme épistémologique. Persuadée que l'être humain ne peut rien attendre d'une nature dont il est une production en creux, une béance d'être ${ }^{1}$ - puisque celle-ci a oublié de le prémunir contre les dangers qui inéluctablement le guettent en le dotant des moyens physiques appropriés - la sophistique décide que chacun est en droit de juger du bien-fondé de sa propre éthique comportementale. Chacun doit donc agir avec efficacité en vue d'assurer ses propres intérêts, c'est-à-dire prioritairement sa survie au milieu d'un monde tissé de passions et d'intérêts le plus souvent contradictoires. C'est ainsi que les Sophistes démontrent avec éclat à quel point l'obtention de la puissance est inhérente à la maîtrise du langage, ellemême dérivée du réaménagement des règles qui fournissent le substrat du dispositif communicationnel du discours.

L'analyse sophistique des règles de production du vrai et du vraisemblable s'avère, à bien des égards, précieuse. Le vrai varie immanquablement en fonction des cultures et des modèles d'intelligibilité qu'elles mettent en oeuvre. On oublie trop vite que l'aléthéia grecque n'a que peu de points communs avec la ratio latine. La vérité en ce premier sens se confond avec la réalité, s'opposant au faux-semblant, à l'apparence trompeuse. La ratio latine ne se préoccupe pas de désigner le réel : elle ne fait qu'exprimer l'ordre dont les phénomènes doivent rendre compte. Le premier sens est ontologique, le second est pratique. La difficulté pour nous provient de ce que notre appréhension culturelle moderne et occidentale du vrai résulte de ces deux origines différentes. Aussi par vérité, entendons-nous à la fois ce qui est en réalité, et ce qui est rationnel ${ }^{2}$.

Le savoir mathématique a opéré une nécessaire césure entre les deux concepts, l'un cessant d'être le critère de l'autre. Un ens mathématicum fonctionnant à l'intérieur d'une relation logique peut, à juste titre, être considéré comme vérace. Pour autant cette véracité n'implique pas une connaissance conforme à l'état de la réalité. La recherche

1 Cette définition de l'humain comme néant de l'être constituera l'un des thèmes majeurs de l'oeuvre de J.-P. Sartre. Ce fut aussi l'une des thèses essentielles du jeune Pic de La Mirandole quelques siècles plus tôt.

2 On pense ici à la tentative hégelienne de réconciliation de l'aletheia grecque et de la ratio latine, dans l'assertion selon laquelle réalité et rationalité se confondent, cf. La Raison dans l'Histoire, UGE, 1965. 
éperdue et salvatrice du vrai s'avère implacablement vouée à l'échec ; elle revêt une dimension de dérision tragique qui, malgré les préventions théoriques, est le véritable ressort de toute relation intersubjective. La sophistique illustre originalement la démarche d'abandon de toute prétention de validation par le Vrai. En ignorant le principe d'identité dans le langage, c'est-à-dire l'homologie ${ }^{1}$, elle introduit dans la pratique communicationnelle le relativisme dont l'ensemble de ses perspectives procèdent. Puisque tous les faires sont possibles, alors tous les dires le sont en même part, et inversement. Dans un deuxième temps, toutes les représentations et toutes les opinions sont également vraies, puisque tout ce qui est objet de représentations ou d'opinions pour un sujet quelconque se voit immédiatement attribuer une authenticité relative à lui. A la figure hiératique de la Vérité universelle et totalisante se substitue alors un ensemble mobile de croyances communes qui définissent les contextes pragmatiques de l'analyse des énoncés, faisant basculer par là-même la vérité dans la doxa. C'est la raison pour laquelle la sophistique manifesta un intérêt si vif envers les mécanismes de production du vraisemblable. La question était de savoir quels seraient les moyens, autrement dit les arte facts, qui permettraient de conférer à un énoncé l'apparence du vrai, c'est-à-dire d'emporter l'adhésion, de persuader. Le sophisme devient alors l'arme de la toute-puissance argumentative, jouant avec les apparences d'une logique dont il contribue activement à miner les fondations. Dans l'espace du sophisme, c'est la dimension instrumentale de la communication qui se déploie, marquée par une rhétorique manipulatoire redoutablement efficace.

En développant l'éristique, autrement dit l'art de la disputatio ou débat contradictoire, les Sophistes ont contribué à mettre en lumière la dimension pragmatique de la communication, et mieux encore sa nature essentielle, selon eux. Il s'agit ici en effet de livrer une lutte originaire dont l'enjeu est de provoquer chez l'autre la certitude qu'il est dans l'erreur et qu'il doit donc se soumettre au point de vue du «vainqueur» en se conformant au point de vue de ce dernier, ou bien en se ralliant à sa thèse. Dans cette perspective, le contenu du discours ne revêt aucune importance ; sa forme seule est l'objet de toutes les attentions visant un but unique : emporter l'adhésion.

1 Lire sur ce sujet le dialogue platonicien établissant la liste des outils anti-sophistique : «L Cratyle. De la rectitude des mots». 
Aristote aura beau jeu de déplorer le fait que :

Faire que l'argument le plus faible soit le plus fort, c'est de cela qu'il s'agit. Voilà pourquoi on s'indignait, à bon droit, de la profession de Protagoras, car il s'agit d'une tromperie, d'une non vérité, qui n'est rien en fait, qu'une apparence de vraisemblance qu'on ne rencontre dans aucun art, si ce n'est dans la rhétorique et dans l'éristique ${ }^{1}$.

Humaine, trop humaine, relativiste et utilitaire, la sophistique en attachant davantage de prix à l'apparence du vrai plutôt qu'au vrai lui-même défend avec fermeté une approche originale de la communication, aux antipodes des exigences éthiques et épistémologiques d'un Platon, puis bien plus tard d'un Habermas. Sa définition même de la communication est unique puisqu'elle définit celle-ci avant tout comme l'instrument d'une reconnaissance sociale qui s'obtient non par les voies étroites d'un savoir lentement édifié, mais par le jeu miroitant des apparences disposées avec art. Ainsi ce seul savoir, considéré par les meilleurs esprits du temps comme infime et parcellaire au regard de tous les autres, se dilate-t-il aux dimensions d'un savoir universel. Etre capable de donner l'illusion de posséder toutes les connaissances possibles, c'est cela qui est vraiment les posséder toutes, puisque l'apparence est cela même qui assure la cohésion des individus dans le corps social. On voit ici se mettrent en place de nouveaux mécanismes de cognition où comprendre et être persuadé s'équivalent, où dispenser les formes du savoir et séduire ne font qu'un.

En conséquence de quoi, cette persuasion qui est au discours ce que la séduction est aux actes, exprime l'idée, indubitablement originale, qu'il existe une vérité du non vrai et une non vérité du vrai, directement issues du relativisme. Les Sophistes en effet ne sont pas des nihilistes : en ce sens ils ne professent pas l'idée que tout est faux, mais à l'opposé que tout est vrai, autrement dit que toutes les thèses s'équivalent ${ }^{2}$. Si par conséquent, il est possible de tenir aucun discours pour vrai, c'est parce que ce qui est déclaré pour vrai par le sujet $\mathrm{A}$, peut en même temps se révéler immédiatement faux sous le rapport du sujet $\mathrm{B}$ et inversement. Ravalés au rang de pures conventions, les lois, les savoirs et les vérités

\footnotetext{
Aristote, Rhétorique, Les Belles-Lettres, coll. Guillaume Budé, 1938.

Ce thème de l'incommensurabilité des théories entre elles, sera repris par le critique des sciences Paul Feyerabend dans son Contre la méthode, Point-Seuil, 1988
} 
admises doivent être mises au nombre des idées reçues et acceptées comme telles par la communauté humaine qui se place en connaissance de cause sous leur tutelle normative.

Au problème de la subjectivité du vrai, s'ajoute celui de sa précarité - est vrai aujourd'hui ce qui sera décrété faux demain - ainsi que celui de sa localisation, redécouvert par Pascal - est vrai ici ce qui est faux là-bas. C'est ainsi que se dégage

le caractère de profonde indécidabilité du contenu propositionnel de A ou B sous le rapport de la vérité. C'est alors que prend tout son sens la notion essentielle de kairos, idée selon laquelle l'instant opportun, l'occasion, doit être saisie qui fait fond à l'action et lui confère un substrat et une profondeur dont l'inanité du réel la prive impitoyablement. En poussant jusqu' au bout la logique du relatif et du vraisemblable contre celle de l'Absolu et du Vrai, la sophistique désigne à notre attention certaines caractéristiques indissociables de la pratique communicationnelle et du processus de construction des connaissances. En mettant en valeur l'idée d'utilité, d'efficacité, et de recherche de la maîtrise du rapport intersubjectif mis en perspective par l'opportunité, une telle démarche permet de montrer que le savoir est toujours enchâssé dans un processus culturel d'assujettissement et de domination se déployant au sein d'une structure de pouvoir qui lui est sous-jacente.

L'approche sophistique constitue un moment essentiel de notre histoire collective, bousculant les systèmes fondés sur l'ordre et la stabilité, aiguillonnant l'esprit et le poussant vers une ingéniosité et une insolence sans cesse accrue. A la fois théorie et pratique communicationnelle, elle possède au plus haut point cette richesse plastique d'improvisation et d'invention qui la constitue en une sémiosis unique, préfigurant les formes contemporaines de notre modernité. A les lire, à les relire, on pense à ce mot-programme de Nietzsche, fervent lecteur des Sophistes : "Il faut émietter l'univers, perdre le respect du Tout ». 